\title{
THE VÁZQUEZ MAXIMUM PRINCIPLE AND THE LANDIS CONJECTURE FOR ELLIPTIC PDE WITH UNBOUNDED COEFFICIENTS
}

\author{
BOYAN SIRAKOV AND PHILIPPE SOUPLET
}

\begin{abstract}
We develop a new, unified approach to the following two classical questions on elliptic PDE:

- the strong maximum principle for equations with non-Lipschitz nonlinearities,

- the at most exponential decay of solutions in the whole space or exterior domains. Our results apply to divergence and nondivergence operators with locally unbounded lowerorder coefficients, in a number of situations where all previous results required bounded ingredients. Our approach, which allows for relatively simple and short proofs, is based on a (weak) Harnack inequality with optimal dependence of the constants in the lower-order terms of the equation and the size of the domain, which we establish.
\end{abstract}

\section{INTRODUCTION}

1.1. The setting. Let $\Omega \subseteq \mathbb{R}^{n}, n \geq 2$, be an arbitrary domain in which is given a realvalued uniformly elliptic second order operator, either in divergence form

$$
\mathcal{L}_{D}[u]:=\operatorname{div}\left(A(x) D u+b_{1}(x) u\right)+b_{2}(x) \cdot D u+c(x) u,
$$

or in non-divergence form

$$
\mathcal{L}_{N D}[u]:=\operatorname{tr}\left(A(x) D^{2} u\right)+b_{1}(x) \cdot D u+c(x) u,
$$

or more generally a fully nonlinear Hamilton-Jacobi-Bellman operator (i.e. a supremum or an infimum of operators as in (1.2) ), for instance, an extremal operator of Pucci type

$$
F[u]:=\mathcal{M}_{\lambda, \Lambda}^{ \pm}\left(D^{2} u\right) \pm b(x)|D u|+c(x) u .
$$

Let $\mathcal{L}[u]$ denote any of (1.1)-(1.3). We always assume that $A(x) \in L^{\infty}(\Omega)$ satisfies

(1.4) there exist $0<\lambda \leq \Lambda$ such that $\lambda I \leq A(x) \leq \Lambda I, \quad x \in \Omega ; \quad A \in C(\Omega)$ if $\mathcal{L}=\mathcal{L}_{N D}$.

The lower-order coefficients belong locally to Lebesgue spaces which make possible for weak solutions to satisfy the maximum principle and the Harnack inequality; specifically,

$$
\begin{gathered}
b, b_{1}, b_{2} \in L_{\text {loc }}^{q}(\Omega) \text { for some } q>n, \quad c \in L_{\text {loc }}^{p}(\Omega) \text { for some } p>p_{0}, \text { where } \\
\qquad p_{0}= \begin{cases}n / 2, & \text { if } \mathcal{L}=\mathcal{L}_{D} \\
p_{E}, & \text { if } \mathcal{L}=\mathcal{L}_{N D} \text { or } F\end{cases}
\end{gathered}
$$

and $p_{E}=p_{E}(n, \lambda, \Lambda) \in(n / 2, n)$ is the constant from [18, [8, Theorem 1.9]. In the following $\|b\|$ may denote $\left\|b_{1}\right\|$, or $\left\|b_{1}\right\|+\left\|b_{2}\right\|$, depending on the operator we consider. Also, by "(sub-, super-) solution" we mean that: (i) $u \in H_{\text {loc }}^{1}(\Omega)$ in the case of $\mathcal{L}_{D}$, and $u$ satisfies the (in)equality in the usual Sobolev sense (see [22, Chapter 8]); (ii) $u \in C(\Omega)$ in the case of $\mathcal{L}_{N D}$ or $F$, and $u$ satisfies the (in)equality in the $L^{q}$-viscosity sense (see [9]).

We study the following two classical questions. 
- (Vázquez strong maximum principle) Can the strong maximum principle hold for nonnegative solutions of $\mathcal{L}[u] \leq f(u)$ if $f$ is not Lipschitz ?

- (Landis conjecture) Is it true that solutions of $\mathcal{L}[u]=0$ in $\mathbb{R}^{n}$ or in an exterior domain cannot decay super-exponentially at infinity?

In spite of the extensive research in recent years, many open problems subsist (details will be given below). For instance, answers are almost entirely unavailable for operators with unbounded coefficients.

To our knowledge, no connection between these two problems has been observed before. Here we present a new approach which unifies their treatment, and has the following main advantages.

- It gives answers for operators with (even locally) unbounded lower-order coefficients, in a number of situations where all previous results required bounded ingredients.

- It extends many of the already available results on the Landis conjecture, even for equations with bounded coefficients; in particular, it proves the Landis conjecture for coercive fully nonlinear equations, a question which was completely open.

- It treats simultaneously equations in divergence and non-divergence form, and provides rather short proofs.

The main tools of our method are the weak and the full Harnack inequalities, with optimal dependence of their constants in the lower-order terms and the size of the domain, which we establish in Section 2 .

1.2. A Vázquez type strong maximum principle. A well-known result by Vázquez [48. asserts that any nonnegative classical supersolution of

$$
\Delta u \leq f(u)
$$

in a domain $\Omega$ satisfies the strong maximum principle (SMP), i.e. either $u \equiv 0$ or $u>0$ in $\Omega$, provided $f(0)=0, f \geq 0$ is nondecreasing on $(0, \infty)$, and $f$ satisfies the (sharp) condition:

$$
\int_{0}(F(s))^{-1 / 2} d s=\infty, \quad F(s)=\int_{0}^{s} f(t) d t .
$$

If $f$ has at most linear growth at zero, this is the standard SMP, but (1.8) allows for non-Lipschitz nonlinearities, the most important and representative example being

$$
f(s)=s|\ln s|^{a}, \quad a \leq 2 .
$$

There has been a huge amount of work on extending the Vázquez maximum principle to more general operators in (1.7) and weak solutions, with extensions to quasilinear, fully nonlinear, singular, degenerate elliptic operators, see for instance [42, [41, [20, 21], 40], and the references therein. We refer to the book [41] for a very thorough presentation of this important extension of the SMP. Among many other things, Pucci and Serrin showed that it is sufficient that $f$ be nondecreasing only in a right neighborhood of zero (this type of extension is sometimes referred to in the literature as the Pucci-Serrin maximum principle). They showed also that condition (1.8) is necessary for the strong maximum principle.

Almost all proofs of Vázquez type SMPs use the classical procedure of first proving a Hopf lemma by solving a radial problem. More specifically, thanks to the boundedness of the coefficients one can write an ODE whose solutions provide subsolutions of the given PDE in an annulus, with non-vanishing normal derivatives on the boundary. Then simple comparison provides the results - this strategy has been applied in all above quoted works. An exception is the paper [27] on the pure second order equation $\operatorname{div}(A(x) D u)=f(u)$, 
where measure-theoretic approach to the Harnack inequality is employed, in the style of [12], to get an ODE on the volumes of super-level sets of the solutions. A priori bounds for supersolutions of general equations with bounded coefficients can be found in 32 .

A situation in which the radial/ODE approach does not seem to work is when the equation $\mathcal{L}[u] \leq f(u)$ has unbounded coefficients, and this case has been completely open up to now. The following theorem settles it for nonlinearities as in (1.9).

Theorem 1.1. Assume (1.4)-(1.6). Let u be a nonnegative weak supersolution of

$$
\mathcal{L}[u] \leq f(u) \quad \text { in } \Omega,
$$

where $f$ is continuous on $[0, \infty), f(0)=0$, and

$$
\limsup _{s \rightarrow 0} \frac{f(s)}{s(\ln s)^{2}}<\infty .
$$

If $\operatorname{essinf}_{B} u=0$ for some ball $B \subset \subset \Omega$ then $u \equiv 0$ in $\Omega$.

To our knowledge, this is the first result on SMP for equations with unbounded coefficients and non-Lipschitz nonlinearities. In addition, we do not have any condition of monotonicity of $f$ in a right neighborhood of zero.

In the recent work [39] on solvability of general non-coercive fully nonlinear equations with quadratic dependence in the gradient, all results had to be restricted to bounded coefficients precisely because of the lack of a SMP of Vázquez type for the nonlinearity $f(s)=s|\ln s|$ and lower-order coefficients in $L^{q}, q>n$, which is the natural integrability for the framework of [39] (see the end of [39, Section 2]). So Theorem 1.1 extends all results from [39] to unbounded coefficients.

Let us sketch the main point of the proof of Theorem 1.1, assuming for simplicity $f(s)=$ $s|\ln s|^{a}, a \leq 2$, and $u$ continuous. Since the zeroset of $u$ is closed, it suffices to show that it is also open. If $u$ vanishes at some point, say 0 , then, for each small $\delta, r>0$, the rescaled function $v=u(r x)+\delta$ satisfies $v(0)=\delta$ and $v$ is a positive solution of a linear equation whose main zero order term is of the form $d(x) v$ with $d(x)=r^{2} f(u(r x)) /(u(r x)+\delta)$. By the (weak) Harnack inequality with sharp dependence on the zero order coeffcient, we can estimate the integral of a suitable power of $v$ over the unit ball $B_{1}$ by the quantity

$$
N_{\delta}=v(0) \exp \left[C\|d\|_{\infty}^{1 / 2}\right]=\delta \exp \left\{C r\left\||\ln (u)|^{a / 2} \sqrt{u /(u+\delta)}\right\|_{\infty}\right\},
$$

where the sup norm is taken on $B_{2}$. But it can be seen that $\left\||\ln (u)|^{a / 2} \sqrt{u /(u+\delta)}\right\|_{\infty}=$ $O\left[\ln ^{a / 2}(1 / \delta)\right]$ as $\delta \rightarrow 0$. When $a \leq 2$, by choosing $r>0$ small enough, it follows that $N_{\delta}$ goes to 0 as $\delta \rightarrow 0$; hence $u$ vanishes in a neighborhood of 0 and the zeroset of $u$ is open.

It is an open problem whether (1.11) can be replaced by (1.8) in Theorem 1.1. We remark that (1.8) is a typical ODE hypothesis, while it seems difficult to find an ODE argument in the presence of unbounded coefficients, as we explained above.

1.3. The Landis conjecture. In [37, among many other things, Kondratiev and Landis asked whether a solution of a uniformly elliptic PDE with bounded coefficients in an exterior domain must necessarily be trivial, provided that it decays as $|x| \rightarrow \infty$ more rapidly than $\exp \left(-C_{0}|x|\right)$ for a sufficiently large constant $C_{0}$. This property is known as "the Landis conjecture", also in its sharper form where the optimal $C_{0}$ is sought for, or in a weaker form brought up by Kenig in [29], where the decay to rule out is $\exp \left(-|x|^{1+\epsilon}\right), \epsilon>0$. Landis conjecture can also refer to entire solutions (i.e. defined in the whole space). 
The Landis conjecture has a long history, in particular in the case $n=2$. Meshkov disproved it for complex potentials $c(x)$ and entire complex solutions of $\Delta u+c(x) u=$ 0 , showing the optimal decay to be $\exp \left(-|x|^{4 / 3}\right)$. Important quantitative extensions of Meshkov's result, as well as extensions to more general equations, can be found in [7], [19, [29], [16], [13]. All these works use Carleman type estimates, which do not distinguish between real and complex solutions.

The Landis conjecture is still open for equations with bounded real coefficients, even for entire solutions of $\Delta u+c(x) u=0$; however a lot of work has been done in the last years. In [31] Kenig, Silvestre and Wang prove the weak form of the Landis conjecture in $\mathbb{R}^{2}$, for $\mathcal{L}_{D}$ with bounded coefficients and one of the $b_{i}=0$, under the hypothesis that $c(x) \leq 0$; actually they obtain a more precise quantitative bound, saying that within distance one of each point on the sphere $|x|=R$ there is a point at which $|u|$ is at least $\exp \left(-C_{0} R(\log R)\right)$. They also prove a bound in $\exp \left(-C_{0} R(\log R)^{2}\right)$ for solutions in exterior domains of $\mathbb{R}^{2}$. This paper brought a number of generalizations, see [14, [30], [16], and the references therein. All these works are for $n=2$ and equations in divergence form, and make various hypotheses on the lower-order coefficients of $\mathcal{L}_{D}$ which in particular imply that $\mathcal{L}_{D}$ or its dual satisfy the maximum principle on bounded subdomains.

Recently, Rossi [44] established sharp versions of the Landis conjecture for general linear non-divergence form operators with bounded ingredients, either for radial coefficients, or for radial solutions, or under the hypothesis that $\mathcal{L}_{N D}$ satisfies the maximum principle on bounded subdomains and the solution has a sign on the boundary if the latter is not empty. The proof of the non-radial case in [44] relies heavily on the fact that $e^{-M|x|}$ is a subsolution of the operator for sufficiently large $M>0$, a property which holds only if the coefficients of the operator are bounded. Variants of some of the results in 44] are obtained among other things in the earlier paper [1] via probability techniques, and in the recent work [3] via a duality argument (due to M. Pierre).

The only result on the Landis conjecture in the real case that does not make some hypothesis on the coefficients which implies the validity of the maximum principle is the very recently posted paper [38, which settles Kenig's weak form of the Landis conjecture in dimension 2 , for entire solutions of $\Delta u+c(x) u=0$ and a bounded $c(x)$.

In the real coefficients case, unbounded $b_{i}$ (with $c$ bounded) are considered in [30, [15, for divergence form operators and $n=2$ only, under the restrictions that $b_{i}$ are integrable at infinity, i.e. belong to $L^{q}\left(\mathbb{R}^{2}\right), q>2$, and that $|u|$ grows at most like $\exp \left(C_{0}|x|^{\alpha}\right)$ with $\alpha=1-2 / q \in(0,1)$. These rather strong hypotheses lead to a different Landis type result with stronger conclusion, ruling out solutions that decay like $\exp \left(-C_{1}|x|^{\alpha+}\right)$.

Our goal here is to prove the Landis conjecture in $\mathbb{R}^{n}$ in any dimension, for unbounded lower-order coefficients which are only uniformly locally integrable (and thus bounded coefficients are a very particular case), under the hypothesis that the maximum principle holds in any bounded subdomain. We also consider exterior domains.

Our method is completely different from the previous works, and allows for rather short proofs. It permits us to treat simultaneously divergence and non-divergence equations; for the latter we do not know of any previous results with unbounded coefficients. We also consider fully nonlinear equations, for which no previous results are available at all. We use only the sharp form of the weak and full Harnack inequalities together with the comparison principle and the solvability of the Dirichlet problem in bounded domains. 
We recall the definition of uniformly local Lebesgue spaces. If $h \in L_{\text {loc }}^{s}(\bar{\Omega}), 1 \leq s \leq \infty$, we say that $h \in L_{u l}^{s}(\Omega)$ provided the quantity (norm)

$$
\|h\|_{L_{u l}^{s}(\Omega)}:=\sup _{x \in \mathbb{R}^{n}}\|h\|_{L^{s}\left(\Omega \cap B_{1}(x)\right)}
$$

is finite. The spaces $L_{u l}^{s}$ have been used for instance in [28, 23]. Note that $L_{u l}^{s_{2}}(\Omega) \subset L_{u l}^{s_{1}}(\Omega)$ if $1 \leq s_{1} \leq s_{2} \leq \infty, \Omega \subseteq \mathbb{R}^{n}$. Also, we call exterior domain any $\Omega$ such that $B_{r_{1}} \subset \mathbb{R}^{n} \backslash \Omega \subset B_{r_{2}}$ for some $r_{2}>r_{1}>0$. We will not assume any smoothness on $\partial \Omega$.

Theorem 1.2. Let $\Omega=\mathbb{R}^{n}$ or $\Omega$ be an exterior domain. Assume (1.4), $b, b_{1}, b_{2} \in L_{u l}^{q}(\Omega)$, $c \in L_{\text {ul }}^{p}(\Omega)$, with $n<q \leq \infty, p_{0}<p \leq \infty$, and (1.6). Assume also that $\mathcal{L}$ satisfies the maximum principle in each bounded subdomain of $\Omega$. Then there exists a constant $C_{0}=C_{0}(n, p, q, \Lambda / \lambda)$ such that if $u$ is a solution of

$$
\mathcal{L}[u]=0 \text { in } \Omega, \quad \text { with } u \geq 0 \text { on } \partial \Omega \text { or } u \leq 0 \text { on } \partial \Omega \text { (if } \partial \Omega \text { is not empty), }
$$

and

$$
\lim _{|x| \rightarrow \infty} e^{C_{1}|x|}|u(x)|=0, \quad \text { with } C_{1}:=C_{0}\left(1+\|b\|_{L_{u l}^{q}(\Omega)}^{\frac{1}{1-(n / q)}}+\|c\|_{L_{u l}^{p}(\Omega)}^{\frac{1}{2-(n / p)}}\right)
$$

then $u \equiv 0$.

Remark 1.1. (i) We prove this theorem for Hamilton-Jacobi-Bellman operators which include (1.3) and (1.2) as particular cases (see Section 4). Also, assumption (1.14) can be weakened to

$$
\liminf _{R \rightarrow \infty} e^{C_{1} R} \sup _{|x|=R}|u(x)|=0 .
$$

(ii) By definition, $\mathcal{L}_{D}$ satisfies the maximum principle in a domain $G$ if $\mathcal{L}_{D}[u] \leq 0$ in $G$ and $u^{-} \in H_{0}^{1}(G)$ imply $u^{-}=0$ in $G$. In the non-divergence case $F$ satisfies the maximum principle if $F[u] \leq(\geq) 0$ in $G$ (resp. $\left.\mathcal{L}_{N D}[u] \leq(\geq) 0\right)$ and $u \geq(\leq) 0$ on $\partial G$ implies $u \geq(\leq) 0$ in $G$, for each $L^{q}$-viscosity (sub-/super-)solution $u \in C(\bar{G})$.

As for the boundary conditions in (1.13) for the exterior domain case, they are understood in the standard sense. Namely, $u \leq 0$ on $\partial \Omega$ means $(\varphi u)_{+} \in H_{0}^{1}(\Omega)$ for all $0 \leq \varphi \in C_{0}^{\infty}\left(\mathbb{R}^{n}\right)$, if $\mathcal{L}=\mathcal{L}_{D} ; u \in C(\bar{\Omega})$ and $u(x) \leq 0$ for all $x \in \partial \Omega$, if $\mathcal{L}=\mathcal{L}_{N D}$ or $F$.

$W$ also recall that by interior De Giorgi-Moser estimates (see [22, Theorem 8.24]), any solution of $\mathcal{L}_{D}[u]=0$ is (Hölder) continuous in $\Omega$.

(iii) It is classical that the maximum principle is satisfied in a bounded domain by $\mathcal{L}_{N D}$ if $c \leq 0$ and by $\mathcal{L}_{D}$ if $c+\operatorname{div}\left(b_{1}\right) \leq 0$ in the sense of distributions, but this condition is of course far from necessary. Various more general results are available, see for instance 47] for divergence form equations, [5], 6] for linear equations with bounded coefficients, [46. Prop. 3.4] for fully nonlinear equations with unbounded coefficients. It is also well known that the validity of the maximum principle can be related to the positivity of the first eigenvalue of the operator, or to the existence of a strictly positive supersolution. See [10], [5], [43], 2].

Here is the main idea of the proof of Theorem 1.2. Under our assumption that the operator $\mathcal{L}$ satisfies the maximum principle in bounded subdomains, one can first show the existence of a positive solution $\psi$ of $\mathcal{L}[\psi]=0$ in $\Omega$ (cf. Propositions 4.1 and 4.2). Next, from the Harnack inequality with sharp dependence on the size of the domain (and on the coeffcients), we deduce a precise lower exponential bound on the decay of $\psi$ at infinity. 
Then, for a general (possibly sign-changing) solution $u$ of $\mathcal{L}[u]=0$ in $\Omega$, if $|u|$ decays faster than $\psi$ at infinity, one may apply the comparison principle to $\pm u$ and $\delta \psi$ on the intersection of $\Omega$ with a large ball, for each $\delta>0$. Letting $\delta \rightarrow 0$, we conclude that $u$ has to vanish identically.

The rest of the article is organized as follows. Section 2 is devoted to the statement and proof of the sharp, weak and full, Harnack inequalities. Theorems 1.1 and 1.2 are respectively proved in Sections 3 and 4. In the Appendix, for the reader's convenience and in order to supply a full quotable source, we provide a proof of the usual Harnack inequality, under general hypotheses.

\section{ON THE HARNACK INEQUALITY}

We start by recalling the following classical "half-Harnack" inequalities.

Theorem A. Let $\Omega=B_{2}$. Assume (1.4), $b, b_{1}, b_{2} \in L^{q}\left(B_{2}\right), c, g \in L^{p}\left(B_{2}\right)$, with $q>n$, $p>p_{0}$, and (1.6). Suppose $\|b\|_{L^{q}\left(B_{2}\right)} \leq 1,\|c\|_{L^{p}\left(B_{2}\right)} \leq 1$.

- (weak Harnack inequality) There exist constants $\epsilon, C_{0}>0$ depending only on $n, p, q$, $\lambda, \Lambda$, such that if $u \geq 0$ satisfies $\mathcal{L}[u] \leq g$ in $B_{2}$, then

$$
\left(\int_{B_{3 / 2}} u^{\epsilon} d x\right)^{1 / \epsilon} \leq C_{0}\left(\inf _{B_{1}} u+\|g\|_{L^{p}\left(B_{2}\right)}\right) .
$$

- (local maximum principle) For each $\varepsilon>0$, there exists a constant $C_{\varepsilon}>0$ depending only on $n, p, q, \lambda, \Lambda, \varepsilon$, such that, if $u$ satisfies $\mathcal{L}[u] \geq g$ in $B_{2}$, then

$$
\sup _{B_{1}} u \leq C_{\varepsilon}\left(\left(\int_{B_{3 / 2}}|u|^{\varepsilon} d x\right)^{1 / \varepsilon}+\|g\|_{L^{p}\left(B_{2}\right)}\right) .
$$

In this generality, this theorem was proved in [47] for divergence form operators, and in [34], [36] for fully nonlinear operators.

Remark 2.1. If $\mathcal{L}=\mathcal{L}_{D}$ is in divergence form we can add to the right-hand side of the differential inequality a term div $(h)$, for some $h \in L^{q}\left(B_{2}\right)$ (note $\operatorname{div}\left(L^{q}\right) \subset H^{-1}$ if $q>n$ ), adding also $\|h\|_{L^{q}\left(B_{2}\right)}$ to the right-hand side of the inequalities in Theorem $A$.

Remark 2.2. In [34, 36] the results are actually stated for $c=0$, but extension to arbitrary $c \in L^{p}, p>p_{0}$ is rather straightforward. Specifically, $F[u] \leq g$ and $u \geq 0$ imply $\mathcal{M}_{\lambda, \Lambda}^{-}\left(D^{2} u\right)-b|D u|-c^{-} u \leq g$, and because of the sign $c^{-} \geq 0$ for the latter operator the $A B P$ inequality holds without difference with respect to the case $c=0$. On the other hand $F[u] \geq g$ implies $\mathcal{M}_{\lambda, \Lambda}^{+}\left(D^{2} u\right)+b|D u| \geq-c u+g$ and we can treat cu as a right-hand side, through a well-known argument. Nevertheless, since Theorem A plays a pivotal role in our study, for the reader's convenience and in order to supply a full quotable source, we provide a proof in the appendix.

An essential tool in our analysis are the following Harnack type inequalities with sharp dependence in the lower-order coefficients and the size of the domain.

For any $r$ with $n<r \leq \infty$, we set

$$
\beta_{r}=\frac{r}{r-n}=\frac{1}{1-(n / r)}, \quad \gamma_{r}=\frac{r}{2 r-n}=\frac{1}{2-(n / r)}, \quad \beta_{\infty}=1, \quad \gamma_{\infty}=\frac{1}{2},
$$


and we denote by $G_{R} \subset G_{R}^{\prime}, R>2$, either $G_{R}=B_{R}, G_{R}^{\prime}=B_{R+1}$ or $G_{R}=B_{R} \backslash B_{2}, G_{R}^{\prime}=$ $B_{R+1} \backslash B_{1}$.

Theorem 2.1. Let $\Omega=G_{R}^{\prime}$ for some $R>2$. Assume (1.4), $b, b_{1}, b_{2} \in L^{q}\left(G_{R}^{\prime}\right), c, g \in$ $L^{p}\left(G_{R}^{\prime}\right)$, with $n<q \leq \infty, p_{0}<p \leq \infty$, and (1.6). Set

$$
A=A_{R}:=1+\|b\|_{L_{u l}^{q}\left(G_{R}^{\prime}\right)}^{\beta_{q}}+\|c\|_{L_{u l}^{p}\left(G_{R}^{\prime}\right)}^{\gamma_{p}} .
$$

There exist constants $\epsilon, C_{0}>0$ depending only on $n, p, q, \lambda, \Lambda$, such that the following holds.

- (weak Harnack inequality) If $u \geq 0$ satisfies $\mathcal{L}[u] \leq g$ in $G_{R}^{\prime}$, then

$$
\left(\int_{G_{R}} u^{\epsilon} d x\right)^{1 / \epsilon} \leq e^{C_{0} A R}\left(\inf _{G_{R}} u+\|g\|_{L_{u l}^{p}\left(G_{R}^{\prime}\right)}\right) .
$$

- (local maximum principle) If $u$ satisfies $\mathcal{L}[u] \geq g$ in $G_{R}^{\prime}$, then, for each $\varepsilon>0$,

$$
\sup _{G_{R}} u \leq C_{\varepsilon}\left(A^{n / \varepsilon}\left(\int_{G_{R}^{\prime}}|u|^{\varepsilon} d x\right)^{1 / \varepsilon}+\|g\|_{L_{u l}^{p}\left(G_{R}^{\prime}\right)}\right),
$$

for some constant $C_{\varepsilon}>0$ depending only on $n, p, q, \lambda, \Lambda, \varepsilon$.

- (Harnack inequality) If $u \geq 0$ satisfies $\mathcal{L}[u]=g$ in $G_{R}^{\prime}$, then

$$
\sup _{G_{R}} u \leq e^{C_{0} A R}\left(\inf _{G_{R}} u+\|g\|_{L_{u l}^{p}\left(G_{R}^{\prime}\right)}\right) .
$$

Remark 2.3. The optimality of the constant in (2.4), is obvious from the ODE $u^{\prime \prime}-2 b u^{\prime}-$ $c u=0, b, c \in \mathbb{R}^{+}$, with solution $u(x)=e^{D x}, D=b+\sqrt{b^{2}+c}$.

Remark 2.4. In the particular case when the coefficients of the operator are bounded and the operator is in divergence form, the constant in (2.6) appears as a remark without proof after Theorem 8.20 in [22] (with $G_{R}=B_{R}, G_{R}^{\prime}=B_{4 R},\|g\|_{L^{p}\left(B_{4 R}\right)}$ instead of $\left.\|g\|_{L_{u l}^{p}\left(G_{R}^{\prime}\right)}\right)$. We note however that a straightforward examination of the constants in the proofs of Theorems 8.17-8.18 in [22] does not seem to give exactly that dependence in the norms of the coefficients, and a refinement is needed. More specifically, with $R=1$, following the constants in those theorems one gets a Harnack constant which grows like $\nu^{C(n) \sqrt{\nu}}$ as $\nu \rightarrow \infty$ and not $C(n)^{\sqrt{\nu}}$ as we have above ( $\nu$ in $\left[22\right.$ grows like $\|b\|_{\infty}+\sqrt{\|c\|_{\infty}}$ here, see (8.6) in [22], their $d$ is our c). Note this is in accordance with what we find in other articles which use the same technique, for instance [25, Remark 2.2], where the quoted constant is also like $\nu^{\nu}$. We also observe that there is a misprint in [22, Problem 8.3], the correct statement of that Problem is with $\sqrt{\Lambda / \lambda}+\nu R$, and not $\sqrt{\Lambda / \lambda+\nu R}-$ see the previous remark. Finally, in the non-divergence case, an examination of the constants in Safonov's proof of the weak Harnack inequality in [45] yields an exponential in which the $L^{\infty}$-norms of the coefficients are taken to some (possibly large) power.

Remark 2.5. We obtain the dependence in $R$ in Theorem 2.1 not from a rescaling $x \rightarrow x / R$ but from a "Harnack chain" of balls of fixed radius, which leads to a Harnack inequality in which $G_{R}^{\prime} \backslash G_{R}$ has in-radius of order 1 instead of $R$, and whose constants depend on norms of the coefficients in $L_{u l}^{q}\left(G_{R}^{\prime}\right)$ instead of $L^{q}\left(G_{R}^{\prime}\right)$ (note even for constant functions the latter norm degenerates as $R \rightarrow \infty$ while the former does not). 
Remark 2.6. As in Theorem $A$, if $\mathcal{L}=\mathcal{L}_{D}$ is in divergence form we can add to the right-hand side of the differential (in)equalities in Theorem 2.1 a term div( $h$ ), for some $h \in L^{q}\left(G_{R}^{\prime}\right)$, adding also $\|h\|_{L^{q}\left(G_{R}^{\prime}\right)}$ to the right-hand side of (2.4)-(2.6). Furthermore, the proof of the local maximum principle below shows that (2.5) can be replaced by the more precise estimate

$$
\sup _{G_{R}} u \leq C_{0}\left(A^{n / \varepsilon}\|u\|_{L_{u l}^{\varepsilon}\left(G_{R}^{\prime}\right)}+A^{(n / p)-2}\|g\|_{L_{u l}^{p}\left(G_{R}^{\prime}\right)}\right)
$$

(where $\|\cdot\|_{L_{u l}^{\varepsilon}}$ is still defined by (1.12), although it need not be a norm in case $\varepsilon<1$ ).

Proof of Theorem 2.1. In all that follows $C_{0}>0$ depends on $n, p, q, \lambda, \Lambda$, and may change from line to line.

Step 1. Weak Harnack inequality in small balls. Let $x_{0} \in \mathbb{R}^{n}, r_{0}>0$ and set $B=$ $B_{r_{0}}\left(x_{0}\right), B^{\prime}=B_{2 r_{0}}\left(x_{0}\right)$. Assume $b \in L^{q}\left(B^{\prime}\right), c \in L^{p}\left(B^{\prime}\right)$ and

$$
0<r_{0} \leq\left[2+\|b\|_{L^{q}\left(B^{\prime}\right)}^{\beta_{q}}+\|c\|_{L^{p}\left(B^{\prime}\right)}^{\gamma_{p}}\right]^{-1} \in(0,1 / 2] .
$$

If $u \geq 0$ satisfies $\mathcal{L}[u] \leq g$ in $B^{\prime}$, then we have

$$
\left(\int_{B} u^{\epsilon} d x\right)^{1 / \epsilon} \leq C_{0} r_{0}^{n / \epsilon}\left(\inf _{B} u+r_{0}^{2-n / p}\|g\|_{L^{p}\left(B^{\prime}\right)}\right)
$$

Proof of Step 1. Let $v(y)=u\left(x_{0}+r_{0} y\right)$ for $y \in B_{2}$ (hence $\left.x_{0}+r_{0} y \in B^{\prime}\right)$. The function $v$ satisfies $\tilde{\mathcal{L}}[v] \leq \tilde{g}$ in $B_{2}$, where the coefficients of the modified operator $\tilde{\mathcal{L}}$ are $\tilde{A}(y)=A\left(x_{0}+r_{0} y\right), \tilde{b}(y)=r_{0} b\left(x_{0}+r_{0} y\right), \tilde{c}(x)=r_{0}^{2} c\left(x_{0}+r_{0} y\right)$, and $\tilde{g}(x)=r_{0}^{2} g\left(x_{0}+r_{0} y\right)$. We compute

$$
\begin{aligned}
\|\tilde{b}\|_{L^{q}\left(B_{2}\right)} & =r_{0}\left(\int_{|y|<2}\left|b\left(x_{0}+r_{0} y\right)\right|^{q} d y\right)^{1 / q} \\
& =r_{0}^{1-n / q}\left(\int_{\left|x-x_{0}\right|<2 r_{0}}|b(x)|^{q} d x\right)^{1 / q}=r_{0}^{1-n / q}\|b\|_{L^{q}\left(B^{\prime}\right)}
\end{aligned}
$$

and similarly $\|\tilde{c}\|_{L^{p}\left(B_{2}\right)}=r_{0}^{2-n / q}\|c\|_{L^{p}\left(B^{\prime}\right)}$. Hence by (2.8),

$$
\|\tilde{b}\|_{L^{q}\left(B_{2}\right)} \leq 1, \quad\|\tilde{c}\|_{L^{p}\left(B_{2}\right)} \leq 1 .
$$

It follows from Theorem A that

$$
\left(\int_{B_{1}} v^{\epsilon} d y\right)^{1 / \epsilon} \leq C_{0}\left(\inf _{B_{1}} v+\|\tilde{g}\|_{L^{p}\left(B_{2}\right)}\right),
$$

which gives the claim of Step 1, by scaling back to $u$ and $g$.

Step 2. Proof of the weak Harnack inequality (2.4). Set $r_{0}:=(3 A)^{-1}$, where $A=A_{R}$ is defined by (2.3). Set $\tilde{G}_{R}:=G_{R}+B_{r_{0}}$ and denote by $X_{1}, \ldots, X_{m}$ the points of the grid $\left(\frac{r_{0}}{2 \sqrt{n}} \mathbb{Z}\right)^{n} \cap \tilde{G}_{R}$, whose cardinal satisfies

$$
m \leq C_{1}(n)\left(\frac{R}{r_{0}}\right)^{n}=C_{2}(n)(A R)^{n} .
$$

Set $\mathcal{B}_{i}:=B_{r_{0}}\left(X_{i}\right)$. Observe that the $\mathcal{B}_{i}$ cover $G_{R}$ and that $B_{2 r_{0}}\left(X_{i}\right) \subset G_{R}^{\prime}$. It is easy to see that for any $k, \ell \in\{1, \ldots, m\}$, we can connect $X_{k}$ and $X_{\ell}$ with overlapping balls as follows: there exist an integer

$$
d \leq C_{3}(n) \frac{R}{r_{0}}=C_{4}(n) A R
$$


and indices $\ell_{1}, \ldots, \ell_{d} \in\{1, \ldots, m\}$ such that $\ell_{1}=k, \ell_{d}=\ell$ and

$$
\left|\mathcal{B}_{\ell_{i+1}} \cap \mathcal{B}_{\ell_{i}}\right| \geq C_{5}(n) r_{0}^{n}, \quad i=1, \ldots, d-1 .
$$

Since

$$
0<r_{0}=\frac{1}{3}\left[1+\|b\|_{L_{u l}^{q}\left(G_{R}^{\prime}\right)}^{\beta_{q}}+\|c\|_{L_{u l}^{p}\left(G_{R}^{\prime}\right)}^{\gamma_{p}}\right]^{-1} \leq\left[2+\|b\|_{L^{q}\left(B^{\prime}\right)}^{\beta_{q}}+\|c\|_{L^{p}\left(B^{\prime}\right)}^{\gamma_{p}}\right]^{-1}
$$

with $B^{\prime}=B_{2 r_{0}}\left(X_{\ell_{2}}\right)$, we deduce from Step 1 that

$$
\left(\int_{\mathcal{B}_{\ell_{2}}} u^{\epsilon} d x\right)^{1 / \epsilon} \leq C_{0} r_{0}^{n / \epsilon}\left(\inf _{\mathcal{B}_{\ell_{2}}} u+\|g\|_{L^{p}\left(B^{\prime}\right)}\right) .
$$

On the other hand,

$$
\left(\int_{\mathcal{B}_{\ell_{1}}} u^{\epsilon} d x\right)^{1 / \epsilon} \geq\left(\int_{\mathcal{B}_{\ell_{1}} \cap \mathcal{B}_{\ell_{2}}} u^{\epsilon} d x\right)^{1 / \epsilon} \geq\left(\inf _{\mathcal{B}_{\ell_{2}}} u\right)\left|\mathcal{B}_{\ell_{1}} \cap \mathcal{B}_{\ell_{2}}\right|^{1 / \epsilon} \geq\left(C_{5}(n) r_{0}^{n}\right)^{1 / \epsilon}\left(\inf _{\mathcal{B}_{\ell_{2}}} u\right)
$$

hence, by combining the last two inequalities,

$$
\left(\int_{\mathcal{B}_{\ell_{2}}} u^{\epsilon} d x\right)^{1 / \epsilon} \leq C_{0}\left(\left(\int_{\mathcal{B}_{\ell_{1}}} u^{\epsilon} d x\right)^{1 / \epsilon}+\|g\|_{L^{p}\left(B^{\prime}\right)}\right) \leq C_{0}\left(\left(\int_{\mathcal{B}_{\ell_{1}}} u^{\epsilon} d x\right)^{1 / \epsilon}+\|g\|_{L_{u l}^{p}\left(G_{R}^{\prime}\right)}\right) .
$$

Repeating the process, we obtain

$$
\left(\int_{\mathcal{B}_{\ell}} u^{\epsilon} d x\right)^{1 / \epsilon} \leq C_{0}^{d-1}\left(\left(\int_{\mathcal{B}_{k}} u^{\epsilon} d x\right)^{1 / \epsilon}+\|g\|_{L_{u l}^{p}\left(G_{R}^{\prime}\right)}\right) .
$$

Since the $\mathcal{B}_{\ell}$ cover $G_{R}$, by summing over $\ell \in\{1, \ldots, m\}$ we obtain

$$
\left(\int_{G_{R}} u^{\epsilon} d x\right)^{1 / \epsilon} \leq m^{1 / \epsilon} C_{0}^{d}\left(\left(\int_{\mathcal{B}_{k}} u^{\epsilon} d x\right)^{1 / \epsilon}+\|g\|_{L_{u l}^{p}\left(G_{R}^{\prime}\right)}\right) .
$$

Recalling $m \leq C_{2}(n)(A R)^{n}$ and $d \leq C_{4}(n) A R$, we have $m^{1 / \epsilon} C_{0}^{d} \leq e^{C_{0} A R}$ (by readjusting $C_{0}$ as usual). By using Step 1 again we finally get

$$
\left(\int_{G_{R}} u^{\epsilon} d x\right)^{1 / \epsilon} \leq e^{C_{0} A R}\left(\inf _{\mathcal{B}_{k}} u+\|g\|_{L_{u l}^{p}\left(G_{R}^{\prime}\right)}\right), \quad k \in\{1, \ldots, m\},
$$

which implies (2.4) since the $\mathcal{B}_{k}$ cover $G_{R}$.

Step 3. Proof of the local maximum principle (2.5). We take $r_{0}:=(2 A)^{-1}$, where $A=A_{R}$ is defined by (2.3), and choose $x_{0} \in G_{R}$ such that

$$
\sup _{G_{R}} u \leq \sup _{B_{r_{0}}\left(x_{0}\right)} u .
$$

By using the same rescaling as in Step 1, combined with the second part of Theorem A, we obtain

$$
\sup _{B_{1}} v \leq C_{0}\left(\left(\int_{B_{3 / 2}}|v|^{\varepsilon} d y\right)^{1 / \varepsilon}+\|\tilde{g}\|_{L^{p}\left(B_{2}\right)}\right) .
$$


By scaling back to $u$ and $g$, we get

$$
\sup _{B_{r_{0}}\left(x_{0}\right)} u \leq C_{0}\left(r_{0}^{-n / \varepsilon}\left(\int_{B_{3 r_{0} / 2}\left(x_{0}\right)}|u|^{\varepsilon} d x\right)^{1 / \epsilon}+r_{0}^{2-n / p}\|g\|_{L^{p}\left(B_{2 r_{0}}\left(x_{0}\right)\right)}\right),
$$

and (2.7) - hence in particular (2.5) - follows.

Step 4. Proof of (2.6). The Harnack inequality (2.6) is a combination of the weak Harnack inequality (2.4) and the local maximum principle (2.5). Observe that, after rescaling or through a trivial modification of the above steps, we can replace $G_{R}$ in (2.4) by $\tilde{G}_{R}=B_{R+1 / 2}$ if $G_{R}=B_{R}$ (resp. $\tilde{G}_{R}=B_{R+1 / 2} \backslash B_{3 / 2}$ if $G_{R}=B_{R} \backslash B_{2}$ ). Similarly, we can replace $G_{R}^{\prime}$ in (2.5) by $\tilde{G}_{R}$.

\section{Proof of Theorem 1.1}

We start with an elementary technical lemma, which restates the hypothesis of Theorem 1.1 in a more convenient form for the proof of that theorem.

Lemma 3.1. Assume $f:[0, L] \rightarrow \mathbb{R}$ is continuous for some $L>0$, and $f(0)=0$. Then

$$
\limsup _{s \rightarrow 0} \frac{f(s)}{s(\ln s)^{2}}<\infty
$$

if and only if there exists $k>0$ such that

$$
e^{\sqrt{M_{\delta}}}=o\left(\frac{1}{\delta^{k}}\right) \quad \text { as } \delta \rightarrow 0, \quad \text { where } M_{\delta}:=\max _{s \in[0, L]} \frac{f(s)}{s+\delta} .
$$

Proof. Let us check that (3.1) implies (3.2) for some $k>0$. The assumption (3.1) guarantees that $f(u) \leq C u(\ln u)^{2}$ on $[0, L]$ for some constant $C>0$. Assume $\delta<e^{-2}$. Then

$$
\left[s(\ln s)^{2}\right]^{\prime}=(\ln s)^{2}+2 \ln s>0 \quad \text { on } \quad(0, \delta]
$$

hence

$$
\frac{s(\ln s)^{2}}{s+\delta} \leq \frac{\delta(\ln \delta)^{2}}{s+\delta} \leq(\ln \delta)^{2}, \quad s \in(0, \delta]
$$

whereas

$$
\frac{s(\ln s)^{2}}{s+\delta} \leq \max \left\{(\ln \delta)^{2},(\ln L)^{2}\right\}, \quad s \in[\delta, L]
$$

Consequently,

$$
\sqrt{M_{\delta}} \leq \max _{s \in[0, L]} \sqrt{\frac{C s(\ln s)^{2}}{s+\delta}} \leq \sqrt{C}(|\ln \delta|+|\ln L|) .
$$

Choosing any $k>\sqrt{C}$, we conclude that for sufficiently small $\delta>0$ and some $\bar{C}>0$

$$
\delta^{k} e^{\sqrt{M_{\delta}}} \leq \bar{C} \delta^{k-\sqrt{C}} \rightarrow 0, \text { as } \delta \rightarrow 0 .
$$

Conversely, if (3.2) holds, by setting $s=\delta$ in the definition of $M_{\delta}$ we get

$$
\frac{f(\delta)}{2 \delta} \leq M_{\delta} \leq C_{1} k^{2}(\log \delta)^{2} \quad \text { for all } \delta<L,
$$

which implies (3.1).

For the proof of Theorem 1.1 we need the following slight extension of Theorem 2.1. 
Proposition 3.2. Let $\Omega=B_{2}$. Assume (1.4), $b, b_{1}, b_{2}, h \in L^{q}\left(B_{2}\right), g \in L^{p}\left(B_{2}\right), c=c_{1}+c_{2}$, $c_{i} \in L^{p_{i}}\left(B_{2}\right)$, with $n<q \leq \infty$ and $p_{0}<p, p_{i} \leq \infty, i=1,2$, and (1.6). Set

$$
A=2+\|b\|_{L^{q}\left(B_{2}\right)}^{\beta_{q}}+\left\|c_{1}\right\|_{L^{p_{1}\left(B_{2}\right)}}^{\gamma_{p_{1}}}+\left\|c_{2}\right\|_{L^{p_{2}\left(B_{2}\right)}}^{\gamma_{p_{2}}} .
$$

If $u \geq 0$ satisfies $\mathcal{L}_{D}[u] \leq g+\operatorname{div}(h)$, resp. $F[u] \leq g$ in $B_{2}$, then

$$
\left(\int_{B_{1}} u^{\epsilon} d x\right)^{1 / \epsilon} \leq e^{C_{0} A}\left(\inf _{B_{1}} u+\|g\|_{L^{p}\left(B_{2}\right)}+\|h\|_{L^{q}\left(B_{2}\right)}\right) .
$$

The proof of this proposition is essentially the same as Steps 1 and 2 of the proof of Theorem 2.1 (with $R=1$ ), noting that if $\tilde{c}(x)=r^{2} c(r x)$ and $\bar{p}=\min \left\{p_{1}, p_{2}\right\}$ then

$$
\|\tilde{c}\|_{L^{\bar{p}}\left(B_{2}\right)} \leq C(n)\left(r^{2-n / p_{1}}\left\|c_{1}\right\|_{L^{p_{1}\left(B_{2 r}\right)}}+r^{2-n / p_{2}}\left\|c_{2}\right\|_{L^{p_{2}\left(B_{2 r}\right)}}\right) .
$$

We can now give the:

Proof of Theorem 1.1. Assume for contradiction that $u \geq 0$ is a nontrivial supersolution such that $\operatorname{ess~} \inf _{B} u=0$ for some ball $B \subset \subset \Omega$.

Step 1. In this step we will observe that, up to replacing $\Omega$ by a suitable subdomain $\Omega^{\prime}$, we can assume that $u$ is continuous. If $u$ is a viscosity supersolution, this is so by definition. Thus consider the case when $u$ is a weak Sobolev supersolution.

We first claim that there exists a ball $B^{\prime} \subset \subset \Omega$ such that ess $\inf _{B^{\prime}} u=0$ but the trace of $u$ on $\partial B^{\prime}$ does not vanish identically. Assume the contrary and let

$$
E=\left\{a \in \Omega: \underset{B_{\varepsilon}(a)}{\operatorname{essinf}} u=0 \text { for all } \varepsilon \in(0, \rho(a))\right\} \text {, where } \rho(a)=\operatorname{dist}(a, \partial \Omega) .
$$

First note that $E$ is nonempty. Indeed, if $E$ were empty then, for each $a \in \Omega$, there would exist $\sigma(a) \in(0, \rho(a))$ such that $\operatorname{essinf}_{B_{\sigma(a)}(a)} u>0$. But since the compact $\bar{B} \subset \Omega$ can be covered by a finite number of balls $B_{\sigma\left(a_{i}\right)}\left(a_{i}\right)$, this would contradict $\operatorname{ess} \inf _{B} u=0$. Next, it is clear that for each $a \in E$ we have $u=0$ a.e. in $B_{\rho(a)}(a)$ (since otherwise there would exist $\eta \in(0, \rho(a))$ such that the trace of $u$ on $\partial B_{\eta}(a)$ does not vanish identically). It follows that the set $E$ is open. Let $\left(a_{i}\right)$ be a sequence of $E$ with $a_{i} \rightarrow a \in \Omega$ and set $r=\rho(a)$. We have $B\left(a_{i}, r / 2\right) \subset \subset \Omega$ for $i$ large and $a_{i} \in E$, hence $u=0$ a.e. in $B_{r / 2}\left(a_{i}\right)$, by what we just proved. Taking $i$ large enough we deduce that $u=0$ a.e. in $B_{r / 4}(a)$, hence $a \in E$ and $E$ is closed in $\Omega$. Consequently, $E=\Omega$ and $u=0$ a.e. in $\Omega$. This contradiction proves the claim.

Since 0 is a (sub)solution we can find a solution of $\mathcal{L}_{D}[\tilde{u}]=f(\tilde{u})$ in $B^{\prime}$, such that $0 \leq \tilde{u} \leq u$ in $B^{\prime}$ and $\tilde{u}=u$ on $\partial B^{\prime}$. This follows from the general existence theory, see for instance 17. Theorem 4.9]. Note in that theorem it is assumed that $b_{1}, b_{2} \in L^{\infty}$ but what is used is that the map $u \rightarrow \operatorname{div}\left(b_{1} u\right)+b_{2}(x) \cdot D u$ is continuous from $H_{0}^{1}$ to $H^{-1}$, which is true for $b_{1}, b_{2} \in L^{q}, q>n$ (and even for $b_{1}, b_{2} \in L^{n}, n \geq 3$ ), owing to the Sobolev embedding and $\frac{1}{n}+\frac{n-2}{2 n}+\frac{1}{2}=1$. Also, $0 \leq \inf _{B^{\prime}} \tilde{u} \leq \operatorname{essinf}_{B^{\prime}} u=0$ and the trace of $\tilde{u}$ on $\partial B^{\prime}$ does not vanish. Thus, if we can prove the theorem for continuous solutions, we could apply it with $\Omega$ replaced by $B^{\prime}$ and $u$ replaced by $\tilde{u}$ which is (Hölder) continuous by the De Giorgi-Moser theory, a contradiction.

Step 2. Set $K=\{x \in \Omega ; u(x)=0\}$. Since $u$ is continuous, the set $K$ is closed in $\Omega$. It is nonempty by our assumption on the existence of $B$. Pick any $x_{0} \in K$ and assume $x_{0}=0$ without loss of generality. We are going to show that $u$ vanishes in $B_{r_{1}} \subset \Omega$ for some $r_{1}>0$, from which we deduce that $K$ is open in $\Omega$, so $K=\Omega$, and we are done. 
We extend $f(s)=0$ for $s<0$, and set $u_{\delta}=u+\delta, f_{\delta}(s)=f(s-\delta), \delta \in(0,1)$. Fix $r_{0}>0$ such that $B_{r_{0}} \subset \Omega$. Then $u_{\delta}>0$ solves either

$$
\operatorname{div}\left(A(x) D u_{\delta}+b_{1}(x) u_{\delta}\right)+b_{2}(x) \cdot D u_{\delta}+\left(c(x)-\frac{f_{\delta}\left(u_{\delta}\right)}{u_{\delta}}\right) u_{\delta} \leq \delta\left(c(x)+\operatorname{div}\left(b_{1}\right)\right) \text { in } B_{r_{0}}
$$

or, respectively,

$$
\mathcal{M}_{\lambda, \Lambda}^{-}\left(D^{2} u_{\delta}\right)-b(x)\left|D u_{\delta}\right|+\left(c(x)-\frac{f_{\delta}\left(u_{\delta}\right)}{u_{\delta}}\right) u_{\delta} \leq \delta c(x) \text { in } B_{r_{0}} .
$$

Hence for each $r \in\left(0, r_{0} / 2\right]$ the rescaled function $v_{r}(x)=u_{\delta}(r x)$ is such that

$$
\operatorname{div}\left(\tilde{A} D v_{r}+\tilde{b}_{1} v_{r}\right)+\tilde{b}_{2}(x) \cdot D v_{r}+\left(\tilde{c}(x)-r^{2} \frac{f_{\delta}\left(u_{\delta}\right)}{u_{\delta}}\right) v_{r} \leq \delta\left(\tilde{c}(x)+\operatorname{div}\left(\tilde{b}_{1}\right)\right) \text { in } B_{2},
$$

and similarly for (3.4), where $\|\tilde{b}\|_{L^{q}\left(B_{2}\right)} \leq N,\|\tilde{c}\|_{L^{p}\left(B_{2}\right)} \leq N$, for some constant $N$ independent of $r$ and $\delta$ (see for instance Step 1 in the proof of Theorem 2.1, we can take $N$ to be the largest of $\left.\|b\|_{L^{q}\left(B_{r_{0}}\right)},\|c\|_{L^{p}\left(B_{r_{0}}\right)}\right)$.

We now apply Proposition 3.2 to (3.5), with $p_{1}=p, p_{2}=\infty, c_{1}=\tilde{c}, c_{2}=r^{2} \frac{f_{\delta}\left(u_{\delta}\right)}{u_{\delta}}$. This yields $\left(\right.$ recall $v_{r}(0)=u_{\delta}(0)=\delta, M_{\delta}$ is defined in (3.2) $)$

$$
\left(\int_{B_{1}} v_{r}^{\epsilon} d x\right)^{1 / \epsilon} \leq \exp \left[\bar{C}\left(1+r \sup _{[\delta, L+\delta]} \sqrt{\frac{f_{\delta}(s)}{s}}\right)\right]\left(u_{\delta}(0)+\bar{C} \delta\right)=\bar{C} \delta \exp \left[\bar{C} r \sqrt{M_{\delta}}\right],
$$

with $\bar{C}=\bar{C}(n, \lambda, \Lambda, N)$. We next set $r=r_{1}:=\min \left\{r_{0} / 2,(k \bar{C})^{-1}\right\}$, where $k$ is the number from (3.2). Thus

$$
\left(\int_{B_{r_{1}}} u^{\epsilon} d x\right)^{1 / \epsilon} \leq\left(\int_{B_{r_{1}}} u_{\delta}^{\epsilon} d x\right)^{1 / \epsilon}=r_{1}^{n / \epsilon}\left(\int_{B_{1}} v_{r_{1}}^{\epsilon} d x\right)^{1 / \epsilon} \leq C\left[\delta^{k} e^{\sqrt{M_{\delta}}}\right]^{1 / k} .
$$

Letting $\delta \rightarrow 0$ and using (3.2) we deduce $u \equiv 0$ in $B_{r_{1}}$, which is what we wanted to prove.

\section{Proof of Theorem 1.2}

In this section we consider either the divergence form operator

$$
\mathcal{L}_{D}[u]:=\operatorname{div}\left(A(x) D u+b_{1}(x) u\right)+b_{2}(x) \cdot D u+c(x) u,
$$

where $A$ satisfies (1.4), $b_{1}, b_{2} \in L_{u l}^{q}(\Omega), c \in L_{u l}^{p}(\Omega), q>n, p>p_{0}$ with (1.6); or the fully nonlinear operator

$$
F[u]:=F\left(D^{2} u, D u, x\right)+c(x) u
$$

where $F(M, 0, x)$ is continuous in $(M, x), F(M, e, x)$ is convex (or concave) in $(M, e)$, $F(t M, t e, x)=t F(M, e, x)$ for each $t>0$, and

$\mathcal{M}_{\lambda, \Lambda}^{-}\left(M_{1}-M_{2}\right)-b(x)\left|e_{1}-e_{2}\right| \leq F\left(M_{1}, e_{1}, x\right)-F\left(M_{2}, e_{2}, x\right) \leq \mathcal{M}_{\lambda, \Lambda}^{+}\left(M_{1}-M_{2}\right)+b(x)\left|e_{1}-e_{2}\right|$,

$b \in L_{u l}^{q}(\Omega), q>n, c \in L_{u l}^{p}(\Omega), p>p_{0}$ with (1.6). The linear and Pucci operators in (1.2) and (1.3) are particular cases of such $F[u]$. We observe (see [43, Lemma 1.1]), that since $F$ is positively 1-homogeneous and convex (resp. concave) in $(M, e)$,

$$
\begin{aligned}
F\left(M_{1}, e_{1}, x\right)-F\left(M_{2}, e_{2}, x\right) & \leq F\left(M_{1}-M_{2}, e_{1}-e_{2}, x\right) \\
\text { (resp. } F\left(M_{1}, e_{1}, x\right)+F\left(M_{2}, e_{2}, x\right) & \left.\leq F\left(M_{1}+M_{2}, e_{1}+e_{2}, x\right)\right) .
\end{aligned}
$$


We assume that $\Omega=\mathbb{R}^{n}$ or $\Omega$ is an exterior domain such that (without loss) $B_{1} \subset \mathbb{R}^{n} \backslash \Omega \subset$ $B_{2}$. We start by observing that the hypothesis of Theorem 1.2 implies the existence of a positive solution in $\Omega$.

Proposition 4.1. Under the above hypotheses, if $\mathcal{L}_{D}$ satisfies the maximum principle in each bounded subdomain of $\Omega$ then there exists $\psi \in H_{\mathrm{loc}}^{1}(\bar{\Omega})$ such that $\psi>0$ and $\mathcal{L}_{D}[\psi]=0$ in $\Omega$.

Proposition 4.2. Under the above hypotheses, if $F[u]$ satisfies the maximum principle in each bounded subdomain of $\Omega$ then there exists $\psi \in W_{\text {loc }}^{2, p}(\Omega)$ such that $\psi>0$ and $F[\psi]=0$ in $\Omega$.

Proof of Proposition 4.1. Fix a bounded domain $G \subset \Omega$. Under our assumptions on the coefficients, it is standard that the bilinear form associated with $\mathcal{L}_{D}^{(\sigma)}=\mathcal{L}_{D}-\sigma$ is continuous and coercive on $H_{0}^{1}(G)$ for $\sigma$ large enough (see [22, 47]). By Lax-Milgram theorem $\mathcal{L}_{D}^{(\sigma)}$ is a bijection from $H_{0}^{1}(G)$ to $H^{-1}(G)$. The equation $\mathcal{L}_{D}[u]=g+\operatorname{div}(h)$ can be written $\left(I+\sigma\left(\mathcal{L}_{D}^{(\sigma)}\right)^{-1}\right) u=\left(\mathcal{L}_{D}^{(\sigma)}\right)^{-1}(g+\operatorname{div}(h)$, and the Fredholm alternative (observe the inclusion of $H_{0}^{1}(G)$ in $H^{-1}(G)$ is compact) gives, for each $g \in L^{p}(G), h \in L^{q}(G)$, a unique solution of $\mathcal{L}_{D}[u]=g+\operatorname{div}(h)$ in $H_{0}^{1}(G)$, since the maximum principle guarantees that the kernel of $\mathcal{L}_{D}$ is trivial.

In the case when $\Omega$ is an exterior domain as above, we fix a smooth function $\phi$ such that $\phi=0$ for $|x| \leq 2, \phi=1$ for $|x| \geq 3$ and $0 \leq \phi \leq 1$. If $\Omega=\mathbb{R}^{n}$, we just set $\phi=1$. For given $j \geq 2$, let $G_{j}=\Omega \cap B_{j}$, let $v_{j} \in H_{0}^{1}\left(G_{j}\right)$ be the solution of

$$
\mathcal{L}_{D}\left[v_{j}\right]=-\mathcal{L}_{D}[\phi]=-\operatorname{div}\left(A(x) D \phi+b_{1}(x) \phi\right)-\left(b_{2}(x) \cdot D \phi+c(x) \phi\right),
$$

given by the first paragraph and set $u_{j}=v_{j}+\phi$. Then

$$
\mathcal{L}_{D}\left[u_{j}\right]=0 \text { in } G_{j}, \quad u_{j}=0 \text { on } \partial \Omega \text { (if nonempty), and } u_{j}=1 \text { on } \partial B_{j},
$$

so $u_{j}>0$ in $G_{j}$ by the maximum and the strong maximum principles (the latter is a consequence of the weak Harnack inequality). Note that, by global Hölder regularity (see [22, Theorem 8.29]), we have $u_{j} \in C\left(\Omega \cap \bar{B}_{j}\right)$. Fix an open ball $\omega \subset B_{3} \backslash B_{2}$. We renormalize $u_{j}$ by considering $\tilde{u}_{j}=u_{j} /\left\|u_{j}\right\|_{L^{2}(\omega)}(j \geq 3)$, which satisfies $\mathcal{L}_{D}\left[\tilde{u}_{j}\right]=0$ in $G_{j}$ along with $\left\|\tilde{u}_{j}\right\|_{L^{2}(\omega)}=1$.

Take any integer $m \geq 3$. Since $\inf _{\omega} \tilde{u}_{j} \leq|\omega|^{-1 / 2}\left\|\tilde{u}_{j}\right\|_{L^{2}(\omega)}=c(n)$, when $\Omega=\mathbb{R}^{n}$, the Harnack inequality guarantees that

$$
0 \leq \tilde{u}_{j} \leq C_{1}(m) \text { in } G_{m}, \quad j \geq m+2 .
$$

When $\Omega$ is an exterior domain, we deduce from the Harnack inequality that $0 \leq \tilde{u}_{j} \leq C_{1}(m)$ in $B_{m+1} \backslash B_{2}$ for all $j \geq m+2$, and it then follows from (4.6) (with $j$ replaced by $m$ ) and the maximum principle that

$$
0 \leq \tilde{u}_{j} \leq C_{1}(m) u_{m} \text { in } G_{m}, \quad j \geq m+2 .
$$

Now, for $j \geq m+2$, testing the equation $\mathcal{L}_{D}\left[\tilde{u}_{j}\right]=0$ in $G_{j}$ with $\tilde{u}_{j} \theta_{m}^{2}$, where $\theta_{m} \in C_{0}^{\infty}\left(\mathbb{R}^{n}\right)$ is such that $\theta_{m}=1$ for $|x| \leq m / 2$ and $\theta_{m}=0$ for $|x| \geq m$, we get

$$
\int_{G_{j}} \theta_{m}^{2} D \tilde{u}_{j} \cdot A D \tilde{u}_{j}+2 \theta_{m} \tilde{u}_{j} D \theta_{m} \cdot A D \tilde{u}_{j}=\int_{G_{j}} \theta_{m}^{2} \tilde{u}_{j}\left(b_{2}-b_{1}\right) \cdot D \tilde{u}_{j}-\tilde{u}_{j}^{2} b_{1} \cdot D\left(\theta_{m}^{2}\right)+c \tilde{u}_{j}^{2} \theta_{m}^{2} .
$$


Using Young's inequality, (1.4) and then Hölder's inequality, we easily deduce that

$$
\begin{aligned}
\frac{\lambda}{2} \int_{G_{m / 2}}\left|D \tilde{u}_{j}\right|^{2} & \leq C_{2}(m) \int_{G_{m}}\left(1+\left|b_{2}-b_{1}\right|^{2}+\left|b_{1}\right|+|c|\right) \tilde{u}_{j}^{2} \\
& \leq C_{2}(m)\left(1+\left\|\left|b_{2}-b_{1}\right|^{2}+\left|b_{1}\right|+|c|\right\|_{L^{n / 2}\left(G_{m}\right)}\right)\left\|\tilde{u}_{j}\right\|_{L^{2^{*}}\left(G_{m}\right)}^{2} .
\end{aligned}
$$

From our assumptions on the coefficients and (4.8) (resp., (4.7)), it follows that for all $j \geq m+2$,

$$
\left\|\tilde{u}_{j}\right\|_{H^{1}\left(G_{m / 2}\right)} \leq C(m)\left(1+\lambda^{-1 / 2}\right)\left\|u_{m}\right\|_{L^{2^{*}}\left(G_{m}\right)}<\infty \quad\left(\text { resp. }, \leq C(m)\left(1+\lambda^{-1 / 2}\right)\right)
$$

(recall that $u_{m}=v_{m}+\phi \in L^{2^{*}}\left(G_{m}\right)$ due to $v_{m} \in H_{0}^{1}\left(G_{m}\right)$ and Sobolev's imbedding). Therefore, for each $m \geq 3$, the sequence $\left\{\tilde{u}_{j} \theta_{m}\right\}_{j=1}^{\infty}$ is bounded in $H_{0}^{1}(\Omega)$. By a diagonal procedure, we deduce that $\tilde{u}_{j}$ has a subsequence which converges weakly in $H_{l o c}^{1}(\bar{\Omega})$ and strongly in $L_{l o c}^{2}(\bar{\Omega})$ to a nonnegative solution $\psi$ in the whole $\Omega$, and $\psi$ is nontrivial due to $\|\psi\|_{L^{2}(\omega)}=1$. As we recalled in Remark 1.1, $\psi$ is Hölder continuous in $\Omega$. Finally, we have $\psi>0$ by the SMP.

Remark 4.1. Although we shall not use this fact, we note that, in the case when $\Omega$ is an exterior domain, the function $\psi$ obtained in Proposition 4.1 actually satisfies homogeneous boundary conditions, in the sense that $\varphi \psi \in H_{0}^{1}(\Omega)$ for all $\varphi \in C_{0}^{\infty}\left(\mathbb{R}^{n}\right)$.

Proof of Proposition 4.2. Fix a bounded smooth domain $G \subset \Omega$. Since $\left\|(c-\sigma)^{+}\right\|_{L^{p}(G)} \rightarrow 0$ as $\sigma \rightarrow \infty$, we can fix $\sigma$ large enough so that the operator $F[u]-\sigma u$ satisfies the hypotheses of [46. Theorem 1], in particular, condition (6) there. By that theorem and well-known regularity results (see the Remark below) for each $v \in C(\bar{G}), g \in L^{p}(G)$, there is a unique $u \in W_{\text {loc }}^{2, p}(G) \cap C^{\alpha}(\bar{G})$ such that $F[u]-\sigma u=g-\sigma v$ in $G$ and $u=0$ on $\partial G$. The operator $S: C(\bar{G}) \rightarrow C(\bar{G})$ given by $S[v]=u$ is thus well defined and compact.

We briefly recall the Leray-Schauder alternative.

Theorem 4.3 (Corollary 1.19, [4]). Let $S: X \rightarrow X$ be compact, where $X$ is a Banach space. Then one of the following holds:

(i) $x-t S(x)=0$ has a solution for every $t \in[0,1]$, or

(ii) the set $\{x: \exists t \in[0,1]: x-t S(x)=0\}$ is unbounded.

If the alternative (ii) happened for our $S$, we would have a sequence $u_{n}$ such that $\left\|u_{n}\right\|_{C(\bar{G})} \rightarrow \infty$ and $F\left[u_{n}\right]-\sigma u_{n}=t_{n}\left(g-\sigma u_{n}\right)$ in $G, u_{n}=0$ on $\partial G$, for some $t_{n} \in[0,1]$. Setting $\tilde{u}_{n}=u_{n} /\left\|u_{n}\right\|_{C(\bar{G})}$, we see that $\left\|u_{n}\right\|_{C^{\alpha}(\bar{G})} \leq C$, by global Hölder regualrity (see 34, Theorem 6.2]). Passing to the limit along a subsequence, using the stability properties of viscosity solutions with respect to uniform convergence ([34, Proposition 9.4]), we find a solution of $F[\tilde{u}]-\left(1-t_{0}\right) \sigma \tilde{u}=0$ in $G, \tilde{u}=0$ on $\partial G$, and $\tilde{u} \not \equiv 0$, which contradicts the maximum principle. Note $\left(1-t_{0}\right) \sigma \geq 0$ so $F-\left(1-t_{0}\right) \sigma$ satisfies the maximum principle if $F$ does.

So by the Leray-Schauder alternative the equation $F[u]=g$ in $G, u=0$ on $\partial G$, has a solution for each $g \in L^{p}(G)$, which is strong by regularity $\left(u \in W_{\mathrm{loc}}^{2, p}(G) \cap C^{\alpha}(\bar{G})\right)$, and then unique by the maximum principle. We now solve $F[v]=-c$ in $G, v=0$ on $\partial G$, and set $u=v+1$. Then $F[u]=0$ in $G$ and $u=1$ on $\partial G$ so $u>0$ in $\bar{G}$, by the maximum and the strong maximum principle.

Take an increasing sequence of smooth domains $\Omega_{j} \subset \Omega$ which converges to $\Omega$. Set $G_{j}=\Omega_{j} \cap B_{j}$ and take the solutions $u_{j}$ given by the above procedure in $G_{j}$. Fix a point 
$x_{0} \in G_{1}$ and replace $u_{j}$ by $\tilde{u}_{j}=u_{j} / u_{j}\left(x_{0}\right), F\left[\tilde{u}_{j}\right]=0$ in $G_{j}, \tilde{u}_{j}>0$ in $G_{j}, \tilde{u}_{j}\left(x_{0}\right)=1$. By the Harnack inequality, for each compact $K \subset \Omega$, we have $0<u_{j} \leq C(K)$ in $K$, for $j>j_{0}(K)$, where $j_{0}$ is such that $K \subset \Omega_{j_{0}}$. By regularity $u_{j}$ is bounded in $W^{2, p}(K)$, and hence (up to a subsequence) converges weakly in that space and strongly in $C(K)$ (recall $p>n / 2)$. Recalling the stability of viscosity solutions with respect to uniform convergence, and that the operator $u \rightarrow b|D u|+c u$ is precompact from $W^{2, p}$ to $L^{p}$ for $b \in L^{q}, c \in L^{p}$, $q>n, p>n / 2$, by the embeddings $W^{2, p} \hookrightarrow W^{1, n p /(n-p)} \hookrightarrow C^{\alpha}$, we deduce by a diagonal procedure that a subsequence of $u_{j}$ converges weakly in $W_{\text {loc }}^{2, p}(\Omega)$ and strongly in $C_{\text {loc }}(\Omega)$ to a strong solution $\psi$ in $\Omega$, with $\psi\left(x_{0}\right)=1$. Finally, we have $\psi>0$ by the SMP.

Remark 4.2. For the reader's convenience we quote precisely the results from the general theory of fully nonlinear equations with measurable coefficients. The bases of the theory for bounded ingredients can be found in [9], 11, [49]. Extensions to unbounded coefficients, in the generality which we require were obtained as follows: the ABP inequality can be found in [33], the Harnack inequality in [34], [36], the global $C^{\gamma}$ estimates in [34, Theorem 6.2], the strong solvability and $W_{\mathrm{loc}}^{2, p}$-estimates for extremal equations in [34, Theorem 7.1] (the same proof applies to convex/concave operators) the stability of viscosity solutions with respect to uniform convergence in [34, Proposition 9.4], global $W^{2, p}$ estimates follow either from [49] (with a straightforward extension to unbounded coefficients using the already available global $C^{\gamma}$ estimates and stability) or from [35, Proposition 2.4]. Note also that the results in [46] were stated for $c, f \in L^{n}$, however all ingredients of the proofs in [46], which we just quoted, were later established for $c, f \in L^{p}, p>p_{0}$.

Proof of Theorem 1.2. If the elliptic operator is in divergence form, let $\psi$ be the function given by Proposition 4.1 (recall $\psi$ is continuous, see Remark 1.1). In the fully nonlinear case, if $F$ is convex in the derivatives of $u$, let $\psi$ be the function given by Proposition 4.2 applied to $F[u]$; whereas if $F$ is concave let $\psi$ be the function given by Proposition 4.2 applied to $\tilde{F}[u]=-F[-u]$. In all cases, normalize $\psi$ so that $\psi\left(x_{0}\right)=1$ for some point $x_{0} \in \mathbb{R}^{n} \backslash B_{2} \subset \Omega$. Then the sharp Harnack inequality, (2.6) in Theorem 2.1] with $g=0$, gives $\left(G_{R}\right.$ is defined in Theorem 2.1)

$$
\inf _{G_{R}} \psi \geq e^{-C_{1} R} .
$$

Let $u$ be a solution as in Theorem 1.2, with $u \leq 0$ on $\partial \Omega$ if the latter is not empty (or replace $u$ by $-u$, and $F$ by $\tilde{F}$ in the fully nonlinear case). Fix $\delta>0$. Under assumption (1.15) (in particular if (1.14) is true), there exists a sequence $R_{i}>3, R_{i} \rightarrow \infty$ such that

$$
u<\delta \psi \text { on } \partial B_{R_{i}} \text {. }
$$

With $\delta$ and $i$ being fixed, our goal is now to apply the maximum principle to show that $u \leq \delta \psi$ in $\Omega_{i}:=\Omega \cap B_{R_{i}}$. We shall not work directly with $u-\delta \psi$, because difficulties arise in order to verify the boundary conditions on $\partial \Omega$ when $\Omega$ is nonsmooth. It turns out that this can be circumvented by considering instead $z:=u_{+}-\delta \psi$ (in the divergence case) or $\tilde{z}:=(u-\delta \psi)_{+}($in the non-divergence case).

First of all, we observe that

$$
\mathcal{L}_{D}[z] \geq 0 \quad(\operatorname{resp} ., F[\tilde{z}] \geq 0) \quad \text { in } \Omega .
$$

Indeed, in the divergence case, this follows from the fact that $D\left(u_{+}\right)=\chi_{\{u>0\}} D u$, along with Kato's inequality

$$
\operatorname{div}\left(A(x) D u^{+}\right) \geq \chi_{\{u>0\}} \operatorname{div}(A(x) D u)
$$


(in the weak Sobolev sense). In the nondivergence case, (4.10) follows from the fact that the maximum of the two viscosity (sub-) solutions $u-\delta \psi$ and 0 is a viscosity subsolution. To check that $u-\delta \psi$ is a subsolution, i.e. $F[u-\delta \psi] \geq 0$, we use (4.4) if $F$ is convex, resp. (4.5) if $F$ is concave, as well as the choice of the strong solution $\psi$ we made above.

We next turn to the boundary conditions on $\partial \Omega_{i}=\partial \Omega \cup \partial B_{R_{i}}$. First considering the divergence case, we shall check that $z \leq 0$ on $\partial \Omega_{i}$, which means

$$
z_{+} \in H_{0}^{1}\left(\Omega_{i}\right) \text {. }
$$

Since no smoothness is assumed on $\Omega$, we cannot use traces on $\partial \Omega$ and some care is needed. Fix a smooth function $\varphi$ such that $\varphi=1$ for $|x| \leq 2, \varphi=0$ for $|x| \geq 3$ and $0 \leq \varphi \leq 1$. By assumption (cf. Remark 1.1), we have $u^{+} \varphi \in H_{0}^{1}(\Omega)$, hence actually $u^{+} \varphi \in H_{0}^{1}\left(\Omega_{i}\right)$. Therefore there exists a sequence $\theta_{j} \in C_{0}^{\infty}\left(\Omega_{i}\right)$ such that $\theta_{j} \rightarrow u^{+} \varphi$ in $H^{1}(\Omega)$. Now setting $\phi_{j}:=\theta_{j}-\delta \varphi \psi+(1-\varphi)\left(u_{+}-\delta \psi\right)$ and recalling $\psi \in H_{l o c}^{1}(\bar{\Omega})$ and $u \in H_{l o c}^{1}(\Omega)$, we have $\phi_{j} \in H^{1}\left(\Omega_{i}\right)$ with $\phi_{j} \rightarrow u_{+}-\delta \psi=z$, hence $\left(\phi_{j}\right)_{+} \rightarrow z_{+}$, in $H^{1}\left(\Omega_{i}\right)$. But, using (4.9), $\varphi, \psi \geq 0$, and the continuity of $u$ and $\psi$ in $\Omega$ (cf. Remark 1.1), in particular near $\partial B_{R_{i}}$, we easily check that $\left(\phi_{j}\right)_{+} \in H^{1}\left(\Omega_{i}\right)$ vanishes in a neighborhood of $\partial \Omega$, as well as on $\partial B_{R_{i}}$, hence $\left(\phi_{j}\right)_{+} \in H_{0}^{1}\left(\Omega_{i}\right)$. This guarantees (4.11). In the nondivergence case, we need to show that

$$
\tilde{z} \in C\left(\bar{\Omega}_{i}\right) \quad \text { with } \tilde{z} \leq 0 \text { on } \partial \Omega_{i} .
$$

But since $u \in C(\bar{\Omega})$ with $u \leq 0$ on $\partial \Omega$ and $\psi \in C(\Omega)$, property (4.12) follows from (4.9) and the fact that $0 \leq \tilde{z}=(u-\delta \psi)_{+} \leq u$.

Now, in view of (4.10)-(4.12), we deduce from the maximum principle that $z \leq 0$ or $\tilde{z} \leq 0$, hence $u \leq \delta \psi$ in $\Omega \cap B_{R_{i}}$. Letting $i \rightarrow \infty$ and then $\delta \rightarrow 0$ we conclude that $u \leq 0$. But then Theorem 2.1 applies to $-u$, hence, after the normalization $u\left(x_{0}\right)=-1$, either $u \equiv 0$ or $\inf _{G_{R}}|u| \geq e^{-C_{1} R}$ for all $R>2$, a contradiction with assumption (1.15).

\section{Appendix. Proof of Theorem A.}

First, in the divergence case, Theorem A follows from [47] (or see Remark at the end of [22, Section 8.10]). So we concentrate here on the non divergence (fully nonlinear) case.

We recall the $\mathrm{ABP}$ inequality: if $\operatorname{diam}(\Omega) \leq 1,\|b\|_{L^{q}(\Omega)} \leq 1, c, g \in L^{p}(\Omega), p>p_{0}$, $b, c, g \geq 0$, and $w \in C(\bar{\Omega})$ is a viscosity solution of

$$
\mathcal{M}_{\lambda, \Lambda}^{+}\left(D^{2} w\right)+b|D w|-c w \geq-g \quad \text { in } \Omega, \quad u \leq 0 \quad \text { on } \partial \Omega,
$$

then for some $C_{0}=C_{0}(n, q, \lambda, \Lambda)$

$$
\sup _{\Omega} w \leq C_{0}\|g\|_{L^{p}\left(\Omega^{+}\right)}, \quad \Omega^{+}=\{x \in \Omega: w(x)>0\} .
$$

This follows from Theorem 2.9 in [33], applied on each connected component of $\Omega^{+}$(noting that $-c w \leq 0$ on that set).

We now prove Theorem A. We start with the weak Harnack inequality, and provide a proof based on the original approach by Krylov and Safonov, [45]. Note that $F[u] \leq g$, (4.3) and $u \geq 0$ imply

$$
\mathcal{M}_{\lambda, \Lambda}^{-}\left(D^{2} u\right)-b|D u|-c^{-} u \leq g, \quad u \geq 0 .
$$

We assume that (5.1) holds in $B_{2},\|b\|_{L^{q}\left(B_{2}\right)} \leq 1,\|c\|_{L^{p}\left(B_{2}\right)} \leq 1$, and want to prove (2.1). 
In the following all constants will be allowed to depend on $n, p, q, \lambda, \Lambda$. By a simple covering argument, it is enough to show that there exists $\rho_{0} \in(0,1 / 2)$ such that for each $x_{0} \in B_{1}$

$$
\left(\int_{B_{\rho_{0}}\left(x_{0}\right)} u^{\epsilon} d x\right)^{1 / \epsilon} \leq C\left(\inf _{B_{\rho_{0}}\left(x_{0}\right)} u+\|g\|_{L^{p}\left(B_{2 \rho_{0}}\left(x_{0}\right)\right)}\right) .
$$

Proposition 5.1. There exist positive constants $\rho_{0}, \kappa, \delta, \bar{C}$ such that for each $x_{1} \in B_{1}$ and each $\rho \in\left(0, \rho_{0}\right]$, if for some $a>0$

$$
\left|\{u>a\} \cap B_{\rho}\left(x_{1}\right)\right| \geq(1-\delta)\left|B_{\rho}\left(x_{1}\right)\right|
$$

then

$$
\inf _{B_{\rho}\left(x_{1}\right)} u>\kappa a-\bar{C} \rho^{2-n / p}\|g\|_{L^{p}\left(B_{2 \rho}\left(x_{1}\right)\right)} .
$$

Proof. Rescaling $x \rightarrow\left(x-x_{1}\right) / \rho$ we can assume that $u$ is a nonnegative solution of

$$
\mathcal{M}_{\lambda, \Lambda}^{-}\left(D^{2} u\right)-b_{\rho}|D u|-c_{\rho} u \leq g_{\rho}, \quad \text { in } B_{2}
$$

where $b_{\rho}(x)=\rho b\left(x_{1}+\rho x\right), c_{\rho}(x)=\rho^{2} c\left(x_{1}+\rho x\right), g_{\rho}(x)=\rho^{2} g\left(x_{1}+\rho x\right)$ and $\left\|b_{\rho}\right\|_{L^{q}\left(B_{2}\right)}=$ $\rho^{1-n / q}\|b\|_{L^{q}\left(B_{2 \rho}\left(x_{1}\right)\right)} \leq \rho^{1-n / q},\left\|c_{\rho}\right\|_{L^{p}\left(B_{2}\right)} \leq \rho^{2-n / p}$. The choice of $\rho_{0}$ will be made so that these norms be sufficiently small.

Assume first $a=1$ and set $v(x)=1-|x|^{2}$. We have $\left|\{u>1\} \cap B_{1}\right| \geq(1-\delta)\left|B_{1}\right|$, hence in particular

We have in $B_{1}$

$$
\left|\{v>u\} \cap B_{1}\right| \leq \delta\left|B_{1}\right| .
$$

$$
\begin{aligned}
\mathcal{M}_{\lambda, \Lambda}^{+}\left(D^{2}(v-u)\right)+b_{\rho}|D(v-u)|-c_{\rho}(v-u) & \geq \mathcal{M}_{\lambda, \Lambda}^{-}\left(D^{2} v\right)-b_{\rho}|D v|-c_{\rho} v-g_{\rho} \\
& \geq-C\left(1+b_{\rho}+c_{\rho}\right)-g_{\rho},
\end{aligned}
$$

and $v-u \leq 0$ on $\partial B_{1}$, so by the ABP inequality (applied with $p$ substituted by $n$ in case $p>n)$

$$
\sup _{B_{1}}(v-u) \leq C\left(\left|\Omega^{+}\right|^{1 / p}+\left\|b_{\rho}\right\|_{L^{q}\left(B_{2}\right)}+\left\|c_{\rho}\right\|_{L^{p}\left(B_{2}\right)}\right)+C_{0}\left\|g_{\rho}\right\|_{L^{p}\left(B_{2}\right)},
$$

where $\left|\Omega^{+}\right|=|\{v-u>0\}| \leq \delta\left|B_{1}\right|$. By choosing $\delta$ and $\rho_{0}$ sufficiently small we obtain

$$
\sup _{B_{1}}(v-u) \leq \frac{1}{4}+C_{0}\left\|g_{\rho}\right\|_{L^{p}\left(B_{2}\right)}
$$

Since $v \geq 3 / 4$ in $B_{1 / 2}$ we get

$$
u \geq \frac{1}{2}-C_{0}\left\|g_{\rho}\right\|_{L^{p}\left(B_{2}\right)} \quad \text { in } B_{1 / 2} .
$$

For arbitrary $a>0$, by replacing $u$ by $\tilde{u}=u / a$ we get

$$
\tilde{u} \geq \frac{1}{2}-\frac{C_{0}}{a}\left\|g_{\rho}\right\|_{L^{p}\left(B_{2}\right)} \quad \text { in } \quad B_{1 / 2} .
$$

Now, if $a<4 C_{0}\left\|g_{\rho}\right\|_{L^{p}\left(B_{2}\right)}$ the inequality (5.4) trivially holds with the choice $\kappa=1, \bar{C}=4 C_{0}$ (since its right-hand side is negative). If on the other hand $a \geq 4 C_{0}\left\|g_{\rho}\right\|_{L^{p}\left(B_{2}\right)}$ we have $\tilde{u} \geq 1 / 4$ in $B_{1 / 2}$. We then take

$$
w(x)=\frac{1}{4} \frac{|x|^{-s}-2^{-s}}{(1 / 2)^{-s}-2^{-s}} .
$$


where $s>0$ is such that $\mathcal{M}_{\lambda, \Lambda}^{-}\left(D^{2}\left(|x|^{-s}\right)\right)=0$, that is, $\lambda(s+1)=\Lambda(N-1)$. As above

$$
\begin{aligned}
\mathcal{M}_{\lambda, \Lambda}^{+}\left(D^{2}(w-\tilde{u})\right)+b_{\rho}|D(w-\tilde{u})|-c_{\rho}(w-\tilde{u}) & \geq \mathcal{M}_{\lambda, \Lambda}^{-}\left(D^{2} w\right)-b_{\rho}|D w|-c_{\rho} w-g_{\rho} / a \\
& \geq-C\left(b_{\rho}+c_{\rho}\right)-g_{\rho} / a,
\end{aligned}
$$

with $w-\tilde{u} \leq 0$ on $\partial\left(B_{2} \backslash B_{1 / 2}\right)$, so by the $\mathrm{ABP}$ inequality for each $\varepsilon>0$ there exists $\rho_{0}>0$ such that if $\rho \in\left(0, \rho_{0}\right]$

$$
\sup _{B_{2} \backslash B_{1 / 2}}(w-u) \leq \varepsilon-\frac{C_{1}}{a}\left\|g_{\rho}\right\|_{L^{p}\left(B_{2}\right)}
$$

Setting $\varepsilon=\frac{1}{2} \min _{B_{1} \backslash B_{1 / 2}} w$ we obtain

$$
\tilde{u} \geq \frac{\varepsilon}{2}-\frac{C_{1}}{a}\left\|g_{\rho}\right\|_{L^{p}\left(B_{2}\right)} \quad \text { in } \quad B_{1} \backslash B_{1 / 2},
$$

Combining this with (5.6), and choosing $\kappa=\frac{1}{4} \min (1, \varepsilon), \bar{C}=\max \left\{4 C_{0}, C_{1}\right\}$, we deduce that

$$
u>\kappa a-\bar{C}\left\|g_{\rho}\right\|_{L^{p}\left(B_{2}\right)} \text { in } B_{1},
$$

which concludes the proof of Proposition 5.1.

Next we prove (5.2), for the number $\rho_{0}$ given by Proposition [5.1. By replacing $u$ by $\left(\inf _{B_{\rho_{0}}\left(x_{0}\right)} u+\alpha+\|g\|_{L^{p}\left(B_{2 \rho_{0}}\left(x_{0}\right)\right)}\right)^{-1} u$ we see that it is enough to assume that inf $B_{\rho_{0}\left(x_{0}\right)} u \leq 1$, $\|g\|_{L^{p}\left(B_{2 \rho_{0}}\left(x_{0}\right)\right)} \leq 1$, and prove that

$$
\left(\int_{B_{\rho_{0}}\left(x_{0}\right)} u^{\epsilon} d x\right)^{1 / \epsilon} \leq C
$$

with constants independent of $\alpha>0$ (then let $\alpha \rightarrow 0$ ). Recall $\rho_{0} \in(0,1 / 2)$ depends only on $n, p, q, \lambda, \Lambda$.

It follows from Proposition 5.1 that we can find a constant $M>1$ such that

$$
\left|\{u>M\} \cap B_{\rho_{0}}\right| \leq(1-\delta)\left|B_{\rho_{0}}\right|, \quad B_{\rho_{0}}=B_{\rho_{0}}\left(x_{0}\right) .
$$

Indeed, if (5.8) failed, by Proposition 5.1 we would have, setting $M=1+(1 / \kappa)(\bar{C}+2)$, that $u \geq \kappa M-\bar{C} \geq 2$ in $B_{\rho_{0}}$ which is a contradiction with $\inf _{B_{\rho_{0}}} u \leq 1$. Note we also have $\kappa M^{k}-\bar{C} \geq 2 M^{k-1}$, for each $k \geq 1$.

We now apply a well-known argument, to prove by induction that for all $k \in \mathrm{N}, k \geq 1$,

$$
\left|\left\{u>M^{k}\right\} \cap B_{\rho_{0}}\right| \leq(1-c(n) \delta)^{k}\left|B_{\rho_{0}}\right|,
$$

for some (small) constant $c(n)>0$. Specifically, we use the Krylov-Safonov "propagating ink-spots lemma" ([45, Lemma 1.1]), in the form given for instance in [26, Lemma 2.1]:

Lemma 5.2. Let $E \subset F \subset B_{\rho_{0}}$ be open sets. Assume for some $\delta>0$ we have $|E| \leq$ $(1-\delta)\left|B_{\rho_{0}}\right|$, and for any ball $B \subset B_{\rho_{0}}$, if $|B \cap E|>(1-\delta)|B|$ then $B \subset F$. Then $|E| \leq(1-c \delta)|F|$, for some constant $c=c(n)>0$.

The induction proceeds by setting $E=\left\{u>M^{k}\right\} \cap B_{\rho_{0}}, F=\left\{u>M^{k-1}\right\} \cap B_{\rho_{0}}, k \geq 1$. The condition of the lemma is guaranteed by Proposition 5.1 and the choice of $M$ we made.

Then by (5.9) there exists $\varepsilon^{\prime}>0$ such that $\left|\{u \geq t\} \cap B_{\rho_{0}}\right| \leq C \min \left\{1, t^{-\varepsilon^{\prime}}\right\}, t>0$. Indeed, if $t \leq 1$ set $C=\left|B_{\rho_{0}}\right|$. If $t>1$ let $j \in \mathrm{N}$ such that $M^{j-1} \leq t<M^{j}$, so

$$
\left|\{u \geq t\} \cap B_{\rho_{0}}\right| \leq(1-c \delta)^{j-1}\left|B_{\rho_{0}}\right|=M^{-\varepsilon^{\prime}(j-1)}\left|B_{\rho_{0}}\right| \leq M^{\varepsilon^{\prime}}\left|B_{\rho_{0}}\right| t^{-\varepsilon^{\prime}}=\frac{\left|B_{\rho_{0}}\right|}{1-c \delta} t^{-\varepsilon^{\prime}}
$$


if we choose $\varepsilon^{\prime}$ so that $M^{-\varepsilon^{\prime}}=1-c \delta$. Now, take $\epsilon=\varepsilon^{\prime} / 2$, then

$$
\int_{B_{\rho_{0}}} u^{\epsilon}=\frac{\varepsilon^{\prime}}{2} \int_{0}^{\infty} t^{\frac{\varepsilon^{\prime}}{2}-1}\left|\{u \geq t\} \cap B_{\rho_{0}}\right| d t \leq C \int_{0}^{\infty} t^{\frac{\varepsilon^{\prime}}{2}-1} \min \left\{1, t^{-\varepsilon^{\prime}}\right\} d t=C .
$$

In the end we prove the local maximum principle (LMP) in Theorem A. We have $\mathcal{M}_{\lambda, \Lambda}^{+}\left(D^{2} u\right)+b|D u| \geq-c u+f$ so by the already known LMP (see [36]), setting $p_{1}=$ $\left(p+p_{0}\right) / 2>p_{0}$,

$$
\begin{aligned}
\sup _{B_{1}} u & \leq C\left(\left(\int_{B_{5 / 4}}|u|^{\varepsilon} d x\right)^{1 / \varepsilon}+\|c u\|_{L^{p_{1}\left(B_{4 / 3}\right)}}+\|f\|_{\left.L^{p_{1}\left(B_{4} / 3\right.}\right)}\right) \\
& \leq C\left(\left(\int_{B_{5 / 4}}|u|^{\varepsilon} d x\right)^{1 / \varepsilon}+\|u\|_{L^{A}\left(B_{4 / 3}\right)}+\|f\|_{L^{p}\left(B_{4 / 3}\right)}\right),
\end{aligned}
$$

where $1 / A=1 / p_{1}-1 / p$ is given by the Hölder inequality (recall $\|c\|_{L^{p}\left(B_{2}\right)}=1$ ). Finally, we downgrade the $L^{A}$-norm of $u$ on $B_{4 / 3}$ to a " $L^{\varepsilon}$-norm" on $B_{3 / 2}$ through a well-known analysis argument on an expanding sequence of balls (given for instance on pages 74-76 of [24]).

\section{REFERENCES}

[1] Arapostathis A., Biswas A., Ganguly D., Certain Liouville properties of eigenfunctions of elliptic operators, Trans. Amer. Math. Soc. (6) 371 (2019), 4377-4409.

[2] Armstrong S., Principal eigenvalues and an anti-maximum principle for homogeneous fully nonlinear elliptic equations, J. Differential Equations 246 (2009), 2958-2987.

[3] Le Balc'h K., Exponential bounds for gradient of solutions to linear elliptic and parabolic equations, $J$. Funct. Anal. 281 (2021), 109094.

[4] Bandle C., Reichel W., Solutions of quasilinear second-order elliptic boundary value problems via Degree Theory, in Handbook of Differential Equations, Stationary Partial Differential Equations, Chipot, M. and Quittner, P. ed. vol 1, Elsevier, 2004.

[5] Berestycki H., Nirenberg L., Varadhan S., The principal eigenvalue and maximum principle for second order elliptic operators in general domains, Comm. Pure Appl. Math. 47(1994), 47-92.

[6] Berestycki H., Rossi L., Generalizations and properties of the principal eigenvalue of elliptic operators in unbounded domains, Comm. Pure Appl. Math. 68 (2015), 1014-1065.

[7] Bourgain J., Kenig C., On localization in the continuous Anderson-Bernoulli model in higher dimension. Invent. Math. 161 (2005), 389-426.

[8] Cabré X., On the Alexandroff-Bakelman-Pucci estimate and the reversed Hölder inequality for solutions of elliptic and parabolic equations, Comm. Pure Appl. Math. 48, (1995) 539-570 .

[9] Caffarelli L., Crandall M., Kocan M., Świech A., On viscosity solutions of fully nonlinear equations with measurable ingredients, Comm. Pure Appl. Math. 49 (1996), 365-397.

[10] Chicco M., Principio di massimo generalizzato e valutazione del primo autovalore per problemi ellittici del secondo ordine di tipo variazionale, Ann. Mat. Pura Appl. (4) 87 (1970), 1-9.

[11] Crandall M., Kocan M., Swiech, A., $L^{p}$-theory for fully nonlinear uniformly parabolic equations, Comm. Partial Differential Equations 25 (2000), 1997-2053.

[12] Di Benedetto E., Trudinger N., Harnack inequality for quasi-minima of variational integrals, Ann. Inst. H. Poincare Analyse non linéaire 1 (1984), 295-308.

[13] Davey B., Quantitative unique continuation for Schrödinger operators, J. Funct. Anal. 279 (2020), 108566 ,

[14] Davey B., Kenig C., Wang J.-N., The Landis conjecture for variable coefficient second-order elliptic PDEs, Trans. Amer. Math. Soc. 369 (2017), 8209-8237.

[15] Davey B., Wang J.-N., Landis' conjecture for general second order elliptic equations with singular lower order terms in the plane, J. Differential Equations 268 (2020), 977-1042. 
[16] Davey B., Zhu J., Quantitative uniqueness of solutions to second-order elliptic equations with singular lower order terms, Comm. Partial Differential Equations 44 (2019), 1217-1251.

[17] Du Y., Order structure and topological methods in nonlinear PDE, World Scientific, 2006.

[18] Escauriaza L., $W^{2, n}$ a priori estimates for solutions to fully non-linear equations, Indiana Univ. Math. J. 42 (1993), 413-423.

[19] Escauriaza L., Kenig C., Ponce G., Vega L., On uniqueness properties of solutions of Schrödinger equations, Comm. Partial Differential Equations 31 (2006), 1811-1823.

[20] Felmer, P., Montenegro M., Quaas A., A note on the strong maximum principle and the compact support principle, J. Differential Equations 246 (2009), 39-49.

[21] Felmer P., Quaas A., Sirakov B., Solvability of nonlinear elliptic equations with gradient terms, J. Differential Equations 254 (2013), 4327-4346.

[22] Gilbarg D., Trudinger N., Elliptic Partial Differential Equations of Second Order, 2nd edition, Springer Verlag.

[23] Ginibre J., Velo G., The Cauchy problem in local spaces for the complex Ginzburg Landau equation. II. Contraction methods, Comm. Math. Phys. 187 (1997), 45-79.

[24] Han Q., Lin F., Elliptic partial differential equations. Second edition. Courant Lecture Notes in Mathematics, New York; American Mathematical Society, Providence, RI, 2011.

[25] Ignatova M., Kukavica I., Ryzhik L., The Harnack inequality for second-order elliptic equations with divergence-free drifts, Commun. Math. Sci. 12 (2014), 681-694.

[26] Imbert C., Silvestre L., Estimates on elliptic equations that hold only where the gradient is large $J$. Eur. Math. Soc. 18 (2016), 1321-1338.

[27] Julin V., Generalized Harnack inequality for semilinear elliptic equations, J. Math. Pures Appl. (9) 106 (2016), 877-904.

[28] Kato T., The Cauchy problem for quasi-linear symmetric hyperbolic systems, Arch. Rational Mech. Anal. 58 (1975), 181-205.

[29] Kenig C., Some recent applications of unique continuation. Recent developments in nonlinear partial differential equations, 25-56, Contemp. Math., 439, Amer. Math. Soc., Providence, RI, 2007.

[30] Kenig C., Wang J.-N., Quantitative uniqueness estimates for second order elliptic equations with unbounded drift, Math. Res. Lett. 22 (2015), 1159-1175.

[31] Kenig C., Silvestre L., Wang J.-N., On Landis' conjecture in the plane, Comm. Partial Differential Equations 40 (2015), 766-789.

[32] Konkov A., On solutions of quasilinear elliptic inequalities containing terms with lower-order derivatives, Nonlinear Anal. 90 (2013), 121-134.

[33] Koike S., Swiech A., Maximum principle for fully nonlinear equations via the iterated comparison function method, Math. Ann. 339 (2007), 461-484.

[34] Koike S., Swiech A., Weak Harnack inequality for fully nonlinear uniformly elliptic PDE with unbounded ingredients, J. Math. Soc. Japan 61 (3) (2009), 723-755.

[35] Koike S., Swiech A., Existence of strong solutions of Pucci extremal equations with superlinear growth in Du, Journal of Fixed Point Theory and Applications 5, (2009) 291- 304.

[36] Koike S., Swiech A., Local maximum principle for Lp-viscosity solutions of fully nonlinear elliptic PDEs with unbounded coefficients, Commun. Pure Appl. Anal. 11 (2012), 1897-1910

[37] Kondratiev V., Landis E., Qualitative properties of the solutions of a second-order nonlinear equation. (Russian) Mat. Sb. (N.S.) 135 (177) (1988), 346-360, 415; translation in Math. USSR-Sb. 63 (1989), $337-350$

[38] Logunov A., Malinnikova E., Nadirashvili N., Nazarov F., The Landis conjecture on exponential decay, arXiv:2007.07034

[39] Nornberg G., Sirakov B., A priori bounds and multiplicity for fully nonlinear equations with quadratic growth in the gradient, J. Funct. Anal. 276 (2019), 1806-1852.

[40] Pucci P., Radulescu V., The maximum principle with lack of monotonicity, Electron. J. Qual. Theory Differ. Equ. 58 (2018), 1-11.

[41] Pucci P., Serrin J., The maximum principle, Progress in Nonlinear Differential Equations and their Applications, Vol. 73, Birkhäuser, Basel, 2007.

[42] Pucci P., Serrin J., Zou H., A strong maximum principle and a compact support principle for singular elliptic inequalities, J. Math. Pures Appl. 78 (1999), 769-789. 
[43] Quaas A., Sirakov B., Principal eigenvalues and the Dirichlet problem for fully nonlinear elliptic operators, Adv. Math. 218 (2008), 105-135.

[44] Rossi L., The Landis conjecture with sharp rate of decay, Indiana Univ. Math. J. 70 (2021), 301-324.

[45] Safonov M.V., Harnack's inequality for elliptic equations and Hölder property of their solutions. (Russian) Zap. Nauchn. Sem. Leningrad. Otdel. Mat. Inst. Steklov. (LOMI) 96 (1980), 272-287. English transl. in J. Soviet Math, 21 (5) (1983), 851-863.

[46] Sirakov B., Solvability of uniformly elliptic fully nonlinear PDE, Archive for Rational Mechanics and Analysis195 (2010), 579-607.

[47] Trudinger N., Maximum principles for linear, non-uniformly elliptic operators with measurable coefficients, Math. Z. 156 (1977) 291-301.

[48] Vázquez J.L. A strong maximum principle for some quasilinear elliptic equations, Appl. Math. Optim. 12 (1984), 191-202.

[49] Winter N., $W^{2, p}$ and $W^{1, p}$ estimates at the boundary for solutions of fully nonlinear, uniformly elliptic equations, Z. Anal. Anwend. 28 (2009), 129-164. 